\title{
Nanotechnology shows promise for next-generation vaccines in the fight against COVID-19
}

\author{
By Maluta Steven Mufamadi
}

$\mathrm{T}$ he world is currently in a race to create a safe and effective vaccine to control and reduce the spread of the Severe Acute Respiratory Syndrome coronavirus 2 (SARS-CoV-2), the virus that causes the COVID-19 disease. Today they are more than 167 COVID-19 vaccine candidates in development, approximately 138 in preclinical evaluation, and 29 in human clinical trials. ${ }^{1-3}$ However, the vaccines engineered employing traditional approaches such as inactivated and live-attenuated strains of the virus itself are associated with many downsides, including lengthy time to manufacture, high toxicity, and infectiveness. Therefore, there is an urgent need to develop new types of vaccine to address these issues. ${ }^{4}$ Nanotechnology has paved new pathways and provided a new avenue in vaccine development for infectious diseases, such as COVID-19, at an unprecedented speed..$^{5-8}$

Nanovaccines use nanoparticles as adjuvants or carriers of biological moieties such as DNA, RNA, and recombinant proteins to the antigen-presenting cell. ${ }^{6}$ In addition, nanovaccines can boost vaccine effectiveness by inducing both humoral immunity (antibody-mediated immunity)

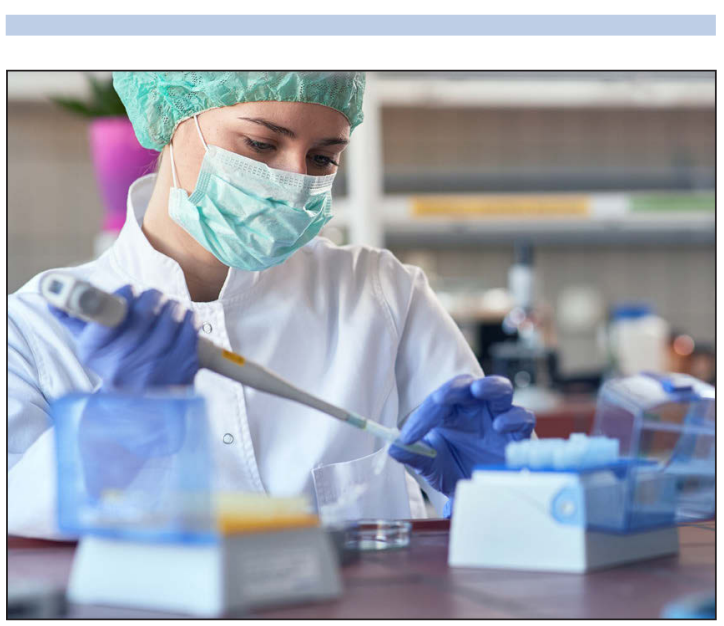

and cell-mediated immune responses. ${ }^{9}$ Nanoparticles based on cationic lipids and synthetic and natural polymer nanomaterials are being explored to enhance the delivery of the active biological moieties in the next generation of vaccine formulations..$^{10}$ These novel nanotechnologies have enabled the next generation of vaccine candidates to enter into human clinical trials.

The impact of nanotechnology in the COVID-19 crisis is seen in the design of effective nanotech-based antiviral disinfectants; the new generation of facemasks that could kill the virus immediately; masks that are washable, reusable, recyclable, and self-sterilized; and rapid COVID-19 diagnostics kits.

\section{Nucleic acid-based vaccines}

Nucleic acid-based vaccines, such as DNA plasmid and messenger RNA (mRNA) vaccines, are among the newest vaccination technologies currently undergoing testing for the fight against COVID-19. DNA plasmid vaccines are based on genetically engineered DNA plasmid containing the DNA sequence encoding the antigen. Messenger RNA vaccines, on the other hand, are made up of mRNA sequence codes for antigen proteins that are identical to or resembling those of the pathogen. ${ }^{11-13}$

Nucleic acid vaccines have many advantages. They are simpler to produce and easy to scale up to large volumes compared to traditional vaccines. DNA and RNA vaccines using synthetic processes require no culture compared to traditional vaccine approaches that require cultures or fermentation processes, which are timeconsuming and expensive.
However, naked DNA plasmid and mRNA-based vaccines are easily eliminated by nucleases through the reticuloendothelial system (RES), which makes it difficult to deliver them into host cells in targeted tissues. ${ }^{14}$ The use of nanoparticles as the vehicle makes it easy to deliver plasmid DNA into the nucleus for the production of encoded antigens or mRNA into the cytoplasm of the targeted host cell to tissues to build immunity against the coronavirus. ${ }^{6}$ The produced virus-like particles can bind to the same receptors as SARS-CoV-2, limiting possible sites for transmission. In addition, it can stimulate the body's adaptive immune system to produce neutralizing antibodies and $\mathrm{T}$ cells against coronaviruses.

\section{RNA nanotech COVID-19 vaccine}

RNA nanotech COVID-19 vaccine candidates use mRNA encoding of the fulllength spike protein of the SARS-CoV-2 to produce a strong immune response against the coronavirus. Lipid nanoparticle (LNP)-encapsulated modified mRNA-based vaccine or mRNA-1273 (currently in a Phase 3 clinical trial) is one of the first RNA nanotech COVID-19 vaccines to reach human trials. ${ }^{14}$ Moderna, Inc., in partnership with the US National Institute of Allergy and Infectious Diseases, developed the mRNA-1273 vaccine. Both preclinical and clinical trial studies are confirming the vaccine to be safe and capable of building immunity against coronaviruses. Other examples of RNA nanotech COVID-19 vaccines include 3LNP-mRNAs BNT162 (Phase 3, BioNTech SE, Fosun Pharma, and Pfizer Inc.), LNP-nCoVsaRNA (Phase 1, Imperial College London), and LNPCureVac - CVnCoV (Phase 2, CureVac Inc., Germany). ${ }^{1,3}$ 


\section{DNA nanotech COVID-19 vaccine}

DNA nanotech COVID-19 vaccine candidates use a small piece of bacterial DNA plasmid that encode the spike protein of SARS-CoV-2 to evoke protective immunity against the coronavirus. ${ }^{6,13}$ Cationic polymeric and LNPs are used to encapsulate plasmid DNA (which has a negative charge) and deliver it into the nucleus of the host cells in targeted tissues (e.g., lung tissues), where it stimulates a specific immune response in the body. The INO-4800 DNA plasmid vaccine (Phase 1/2, DNA coated-PLGA nanoparticles, Inovio Pharmaceuticals, Inc. and the International Vaccine Institute) is an example of a DNA nanotech COVID-19 vaccine that is currently in human clinical trials. ${ }^{15}$ Covigenix is another DNA nanotech vaccine example that is about to enter Phase $1 / 2$ human clinical trials (a DNA vaccine employed using the Fusogenix nanomedicine platform, Entos Pharmaceuticals Inc.). ${ }^{16}$

\section{Recombinant protein nanotech COVID-19 vaccines}

Recombinant protein nanotech COVID-19 vaccine candidates are capable of the delivery of recombinant protein through nanoparticles to the cytoplasm of the targeted host cells. NVX-CoV2373 is an example of a recombinant protein nanotech vaccine to enter into human clinical trials. ${ }^{1,17}$ It was constructed from the full length of the wild-type (naturally occurring, nonmutated strain of a virus) SARS-CoV-2 spike glycoprotein, and the Novavax Inc. recombinant nanoparticle technology was patented with adjuvant Matrix M, a saponin-based adjuvant matrix particle formed by formulating purified saponin from Quillaja saponaria Molina with cholesterol and phospholipid to enhance the immune response. Novavax recombinant nanoparticle technology generated an antigen derived from SARS-CoV-2 spike glycoprotein, which mediates a viral attachment to the human angiotensin-converting enzyme 2 (hACE2) receptors of the host cells for cell entry, which then stimulates the immune system to produce neutralizing antibodies or vaccines. Both preclinical and clinical trials confirmed that NVX-CoV2373, with or without the saponin-based Matrix-M1 adjuvant, is capable of eliciting robust immune responses, an immunoglobulin $\mathrm{G}(\mathrm{IgG})$ anti-spike protein, and high levels of neutralizing antibodies. In addition, the neutralizing antibody responses were found to be four times higher than responses in convalescent serum in patients with COVID-19 who had clinically significant illness. A high level of neutralizing antibodies confirms that the recombinant protein nanotech vaccine may confer protection against the SARS-CoV-2 virus. The human clinical trials also confirmed that NVX-CoV2373 is safe, with mild to moderate side effects and systemic reactogenicity.

\section{Summary}

COVID-19 is possibly a game changer for nanotechnology. There are currently no licensed DNA and mRNA vaccines; therefore, if any of the COVID-19 candidates are successful, this might be the push nanotechnology needs for vaccine development. DNA, mRNA, and recombinant protein delivered into the targeted host cells to build immunity against the coronavirus would not have been feasible without nanoparticles as a delivery vehicle. Nanoparticles provide the solutions to address the unmet delivery challenges associated with the use of naked DNA plasmids or mRNA for vaccine development. Such solutions provide the ability to deliver DNA plasmids or mRNA vaccine in areas previously unreachable. In addition, nanotechnologybased vaccines are easy to design, synthesize, or scale up in larger volume compared to the traditional vaccine approaches (e.g., inactivated and live-attenuated strains). Nanotechnology thus has great potential to be a vital tool for tackling the COVID-19 outbreak and may be a crucial technology to prevent future infectious disease outbreaks.

\section{References}

1. W.M. Detmer, "Coronavirus COVID-19 Vaccines" (2020),https://relief.unboundmedicine.com/ relief/view/Coronavirus-guidelines/2355056/ all/Coronavirus_COVID_19_Vaccines (accessed August 31, 2020)

2. S.P. Kaur, V. Gupta, COVID-19 Vaccine: A Comprehensive Status Report (2020), doi:10.1016/ j.virusres.2020.198114.
3. World Health Organization, "DRAFT Landscape of COVID-19 Candidate Vaccines" (Geneva, Switzerland, 2020).

4. M.D. Shin, S. Shukla, Y.H. Chung, V. Beiss, S.K Chan, O.A. Ortega-Rivera, D.M. Wirth, A. Chen, M. Sack, J.K. Pokorski, N.F. Steinmetz, Nat Nanotechnol. 15 (8), 646 (2020).

5. I. Landau, L. Chard, "News Updates: NanoBased Vaccines and Therapeutics for Treatment of COVID-19" (2020), https://www.nanomedzone. com/covid-19-updates-nano-based-vaccinesfor-treatmentof-covid-19 (accessed August 29 2020).

6. G. Chauhan, M.J. Madou, S. Kaira, V. Chopra, D. Ghosh, S.O. Martinez-Chapa, ACS Nano 14, 7760 (2020)

7. G. Palestino, I. Garcia-Silva, O. Gonzalez-Ortego, S. Rosales-Mendoza, Expert Rev. Anti. Infect. Ther. 18, 849 (2020).

8. StatNano, Nanotechnology in Battle Against Coronavirus (2020), https://statnano.com/nanotechnology-in-battle-against-coronavirus (accessed August 31, 2020)

9. L. Liu, Z. Liu, H. Chen, H. Liu, Q. Gao, F. Cong, G. Gao, Y. Chen, ACS Appl. Bio Mater. 3 (9), 5633 (2020).

10. C. Weiss, M. Carriere, L. Fusco, I. Capua, J.A. Regla-Nava, M. Pasquali, J.A. Scott, F. Vitale, M.A. Unal, C. Mattevi, D. Bedognetti, A. Merkoçi, E. Tasciotti, A. Yilmazer, Y. Gogotsi, F. Stellacci, L.G. Delogu, ACS Nano 14 (6), 6383 (2020).

11. M. Lim, A.Z. Md Badruddoza, J. Firdous, M. Azad A. Mannan, T.A. Al-Hilal, C.-S. Cho, M.A. Islam, Pharmaceutics 12 (1), 30 (2020).

12. L.A. Jackson, E.J. Anderson, N.G. Rouphael, P.C. Roberts, New. Engl. J. Med. (2020), doi:10.1056/ NEJMoa2022483.

13. T.R.F. Smith, A. Patel, K.E. Broderick, Nat Commun. 11 (1), 2601 (2020).

14. K.S. Corbett, B. Flynn, K.E. Foulds, J.R. Francica, New. Engl. J. Med. (2020), doi:10.1056/NEJMoa2024671.

15. R. Carlson, D. Reiter, "INO-4800 DNA Coronavirus Vaccine," Precision Vaccinations (2020), https:// www.precisionvaccinations.com/vaccines/ino4800-dna-coronavirus-vaccine.

16. Biospace. "Entos Pharmaceuticals Announces Selection of Lead DNA Vaccine Candidates for COVID-19 and a \$4.2M Award to Move Forward with Phase I/II Human Trials" (2020) https://www. biospace.com/article/releases/ entos-pharmaceuticals-announces-selectionof-lead-dna-vaccine-candidates-for-covid-19 and-a-4-2m-award-to-move-forward-withphase-i-ii-human-trials/ (accessed September 2, 2020).

17. C. Keech, G. Albert, P. Reed, S. Neal, J.S. Plested, M. Zhu, S. Cloney-Clark, H. Zhou, N. Patel, M.B. Frieman, R.E. Haupt, J. Logue, M. McGrath, S. Weston, P.A. Piedra, I. Cho, A. Robertson, C. Desai, K. Callahan, M. Lewis, P. Price-Abbott, N. Formica, V. Shinde, L. Fries, J.D. Linkliter, P. Griffin, B. Wilkinson, G. Smith, G.M. Gleen, "First-in-Human Trial of a SARS CoV 2 Recombinant Spike Protein Nanoparticle Vaccine," MedRxiv (2020), doi.org/1 0.1101/2020.08.05.20168435. 